\title{
Hacia la búsqueda de una definición de las autoridades administrativas independientes en Francia
}

\section{Towards the search for a definition of independent administrative authorities in France}

Hubert Alcaraz*

\begin{abstract}
Desde finales de los años ochenta se multiplicaron en Francia las autoridades administrativas independientes. Se crearon sin directrices ni programas preestablecidos. Así las cosas, los estatutos se diversificaron, disponiendo para cada autoridad conforme sus especificidades. Desde principios de los años 2000, tanto el legislador como la doctrina, han empezado un trabajo de unificación y de homogeneización de esta categoría, del cual las leyes del 20 de enero de 2017 representan la última etapa. Pero, pese a esos esfuerzos, la categoría sigue buscando su definición y su unidad.
\end{abstract}

Palabras clave: Autoridades administrativas independientes, autoridades públicas independientes, Organización administrativa, Modelo administrativo, Consejo de Estado, Parlamento.
Since the end of the 1980s the authorities multiplied in France independent administrative authorities.

They were created without guidelines or preset program. So statutes have diversified, each authority has its specificities. Since the beginning of the years 2000s, the legislator, as doctrine, have started a work of unification and homogenization of this category, of which the laws of January 20, 2017 represent the last stage. But despite those efforts, the category is still looking for its definition and unit.

Keywords: Independent administrative authorities, independent public authorities, administrative organization, administrative model, council of state, Parliament.

\footnotetext{
* Profesor titular HDR de derecho público en la Universidad de Pau et des pays de I'Adour, miembro del Instituto de estudios ibéricos e iberoamericanos (IE2IA - CNRS UMR 7318). Dirección Postal: Avenue du Doyen R. Poplawski BP 1633-64016 PAU, Francia. Correo electrónico: hubert.alcaraz@univ-pau.fr

Recibido el 17 de enero de 2019 y aceptado el 12 de marzo de 2019.
} 


\section{Introducción}

La cuestión de la regulación administrativa y, en realidad, parte del modelo administrativo francés, acaban de ser objeto de actualización en Francia, puesto que el 20 de enero de 2017 se adoptaron dos leyes con la finalidad de crear un estatus general para las autoridades administrativas independientes y las autoridades públicas independientes. Ello no significa, no obstante, que la figura de la autoridad administrativa independiente o de la agencia autónoma independientes del poder ejecutivo es nueva en el derecho francés, lo cierto es que desde la ley del 6 de enero de 1978, que creó la Comisión Nacional de la Informática y de las Libertades (CNIL), han ido creándose una larga serie de autoridades administrativas independientes. Desde este punto de vista, no cabe duda de que el desarrollo de estas autoridades así como la descentralización administrativa, es una de las mayores evoluciones de la organización de las instituciones administrativas en Francia durante los últimos cincuenta años.

Estas organizaciones constituyen, de hecho, una respuesta institucional a la crisis del modelo administrativo francés. En realidad, la aparición de esas autoridades responde a preocupaciones distintas que son principalmente tres. Primero, la creación de aquellas autoridades debería garantizar a los administrados una protección eficaz de sus libertades y derechos cuando la administración, al ejercer sus competencias, puede especialmente amenazarles. Segundo, el recurso a las autoridades administrativas independientes ha constituido una solución, ya que permitió asegurar una regulación objetiva de los mercados económicos en los cuales participan empresas públicas o que estén susceptibles de ser desajustados por la intervención directa del Gobierno. Es lo que se llama -como se sabe- la regulación económica. En tercer lugar, el crecimiento de casos político-financieros condujo a la adopción de varias medidas destinadas a asegurar la transparencia de la acción pública. Mientras las dos primeras categorías de autoridades siguen desarrollándose, el recurso a aquellos organismos constituyó igualmente un medio para responder a la desconfianza de los administrados respecto de la administración gubernamental.

Sin embargo, desde el punto de vista de la unidad de aquellas autoridades administrativas, ese desarrollo, que fue traducido en su éxito, provocó al mismo tiempo su debilitamiento: ello por tener misiones muy variadas y en sectores muy distintos, a través de formas muy dispares, y la sistematización de sus estatutos parecía difícil. Así, después de un primer informe público del Consejo de Estado en 2001'1 , a partir del $2006^{2}$ fue el Senado quien inició un trabajo con la finalidad de asegurar la racionalización de aquellas autoridades y de organizar un estatus general, antes de que la Asamblea Nacional se preocupara también de la cuestión ${ }^{3}$. La ley orgánica y la ley ordinaria del 20 de enero

\footnotetext{
CONSEIL d'ÉtAT 2001.

2 GÉlard 2006.

3 Dosière et VANNeste 2010.
} 
del 2017, en relación a las autoridades administrativas independientes y las autoridades públicas independientes, emanan precisamente de aquellas labores.

Con la finalidad de profundizar en alguna medida este tema y entender las últimas evoluciones normativas francesas, es necesario observar que la noción de autoridad administrativa independiente en Francia abarca varias entidades: primero, las "autoridades", así designadas en el texto fundador; luego, las autoridades llamadas "autoridades independientes" o "autoridad pública independiente"; en fin, una cierta cantidad de autoridades no cualificadas por los textos, pero que la jurisprudencia y la doctrina acogieron en la categoría general de autoridad administrativa independiente. Sin embargo, desde el origen, es decir, desde principio de la década de los 80, esta diversidad de denominaciones responde a un régimen jurídico heterogéneo que resulta de la yuxtaposición de textos. Para decirlo de otro modo, la ausencia de homogeneidad de la categoría es el reflejo de la diversidad de los estatutos jurídicos de cada una, lo cual se ve reflejado igualmente en sus designios.

Frente a esa diversidad, la independencia de todas esas autoridades puede constituir un elemento de unidad -o por lo menos un punto en común- pero que nace de una pluralidad de disposiciones estatutarias o funcionales; mientras que respecto a sus competencias, también se nota una heterogeneidad, así es que la misma autoridad puede disponer a la vez de atribuciones administrativas clásicas, como el poder de regulación y de atribuciones cuasi jurisdiccionales, así como del poder punitivo y del poder de resolución de conflictos. En todo caso, un elemento de unidad surge de nuevo por el hecho de que todas las autoridades administrativas independientes son objeto de un control que siempre es, al menos, jurisdiccional.

De tal forma que, frente a esas características generales, a un estatuto muy a menudo heterogéneo y un control jurisdiccional que pesa sobre cada una de esas entidades, son dos las observaciones de modo sintético que se pueden hacer para presentar la actualidad de esa figura en el derecho público francés: por un lado, es una noción que sigue siendo excepcional (I); y por otro, es una noción que sigue buscando su unidad (II).

\section{Una noción siempre excepcional}

Hay que reconocerlo: las autoridades administrativas independientes constituyen una curiosidad jurídica. En particular, dos aspectos alimentan la singularidad de las autoridades administrativas independientes, relevando interrogaciones. Para decirlo de otra manera, su carácter excepcional es el resultado, por una parte, de la heterogeneidad de la noción (1) y, por la otra, de esa referencia a su independencia (2).

\section{La heterogeneidad de la noción}

Primero, la originalidad de la categoría "autoridad administrativa independiente" tiene que ver con la definición y la delimitación de esta noción. Res- 
pecto a este tema, por un lado, se puede preguntar sobre si constituye una verdadera categoría jurídica o simplemente una "categoría de hecho". Así, la pregunta sería la siguiente: ¿se trata, en el fondo, de una noción que permite agrupar convenientemente organismos en realidad muy diferentes? ¿० de una noción que puede ver su contenido determinado como concepto, enumerando sus características?

Después del primer organismo calificado como autoridad administrativa independiente -la Comisión Nacional de Informática y Libertades (CNIL)-, creada con la ocasión de la adopción de la ley de 1978 relativa a la informática ${ }^{4}$, organismos similares vieron la luz en diferentes sectores de la vida pública, pero de manera desordenada, es decir, sin directrices ni programas preestablecidos. Hasta el punto de que hoy es difícil proponer una definición general y erigir una lista oficial y exhaustiva de organismos que pertenecen, sin contesta, a aquella categoría.

Esquemáticamente, se oponen dos concepciones: una concepción estrecha y una concepción amplia de la noción de autoridad administrativa:

a) Según una concepción estrecha, se reserva la denominación de autoridad administrativa independiente a un pequeño número de instituciones. La razón sería bastante sencilla en este caso: la autoridad administrativa independiente es tal porque ha sido calificada así por el texto legislativo que la creó o, porque en ausencia de una definición textual, presenta similitudes manifiestas con las primeras.

b) Según una concepción amplia, se puede incluir un número mucho más alto de organismos, por extensión de la cualificación a instituciones que pueden presentar similitudes más o menos importantes con las autoridades administrativas "oficiales". Esta prevalece porque la concepción estrecha sufre de una permisividad legislativa, que hace crecer las denominaciones y hace coexistir las "autoridades administrativas independientes" con las "autoridades públicas independientes", así como las "autoridades independientes" simples. Finalmente, es esta concepción amplia la que domina hoy en día la doctrina, así como en el uso administrativo y político. Y así, se incorpora en la noción de autoridad administrativa independiente, las autoridades públicas independientes y ciertas autoridades no expresamente cualificadas como tales por el texto fundador.

Durante los últimos años, la calificación de "autoridad pública independiente" tuvo bastante éxito y ello se encuentra relacionado con la atribución de la personalidad jurídica otorgada por el legislador. Así es el caso, por ejemplo, de la Autoridad de los Mercados Financieros ${ }^{5}$, la Alta Autoridad de

\footnotetext{
4 Ley No 78-17 de 1978.

5 La Autoridad de los mercados financieros fue creada por la Ley № 2003-706 de 2003 y fusiona el Consejo de los mercados financieros, la Comisión de las operaciones de bolsa y el Consejo de disciplina de la gestión financiera.
} 
Salud ${ }^{6}$, la Agencia Francesa de Lucha contra el Dopaje7, el Alto Consejo de Auditaría de Cuentas ${ }^{8}$, la Alta Autoridad por la Difusión de Obras y la Protección de Derechos en Internet ${ }^{9}$, así como con la Autoridad de Regulación de las Actividades Ferroviarias ${ }^{10}$. Esa evolución, aunque difícilmente puede considerarse ajena al campo de las autoridades administrativas independientes, tal como existen desde principios de los años ochenta, alimenta esa dificultad de definición en la medida en que no hay trabajo de distinción o, al contrario, de fusión de las categorías "autoridades públicas independientes" y "autoridades administrativas independientes". Y a esta primera dificultad hay que añadir también el hecho de que el legislador no siempre se toma la molestia de calificar el organismo que crea, y son los observadores que usan la calificación de autoridad administrativa independiente por algunas características que aparecen en la ley creadora de cada institución.

Básicamente, son tres las características que las definen: primero, la autoridad administrativa independiente tiene el poder de tomar decisiones individuales o reglamentarias en nombre del Estado; segundo, el organismo tiene una verdadera autonomía estatutaria y funcional frente al Estado; y tercero, el organismo no es en sí mismo una jurisdicción cuyas decisiones podrían ser recurridas. Pero, fuera de esos elementos, tenemos que observar cuáles son las incertidumbres y dudas que predominan en la materia. A modo de ejemplo, , con la aparición del Defensor de los derechos (Défenseur des droits), figura que nació de la última reforma constitucional, ${ }^{11}$ es calificado como "autoridad constitucional independiente" no por la Constitución francesa misma ${ }^{12}$ sino por el artículo 2 de su ley orgánica ${ }^{13}$. Pero, lo que sorprende, es que no tiene personalidad jurídica, a diferencia de las autoridades

\footnotetext{
6 Creada por la Ley N²004-810 de 2004.

7 Con la ley N²006-405 de 2006.

8 Instituido por la Ley N²003-706 de 2003.

9 Creada por la Ley N²009-669 de 2009.

10 Creada por la Ley N²009-1503 de 2009; fue transformada en Autoridad de regulación de las actividades ferroviarias y de carretera con la Ley N²015-990 de 2015.

11 Ley Constitucional N²008-724 de 2008.

12 El nuevo artículo 71-1 de la Constitución del 4 de octubre de 1958, insertado por la reforma del 2008, no utiliza la expresión "autoridad constitucional independiente". Solo dispone: "El Defensor de los Derechos velará por el respeto de los derechos y las libertades por parte de las administraciones del Estado, las entidades territoriales, los establecimientos públicos, así como cualquier organismo encargado de una misión de servicio público o respecto del cual la ley orgánica le atribuya competencias. Podrá ser solicitado, en las condiciones previstas en la ley orgánica, por cualquier persona que se considere perjudicada por el funcionamiento de un servicio público o un organismo referido en el primer párrafo. Podrá ser solicitado de oficio. La ley orgánica definirá las atribuciones y las modalidades de intervención del Defensor de los Derechos. Determinará las condiciones en que pueda ser asistido por un colegio para el ejercicio de algunas de sus atribuciones. El Defensor de los Derechos será nombrado por el Presidente de la República por un mandato de seis años no renovable, según el procedimiento previsto en el último párrafo del artículo 13. Sus funciones serán incompatibles con las de miembro del Gobierno y miembro del Parlamento. Las demás incompatibilidades serán fijadas por la ley orgánica. El Defensor de los Derechos dará cuenta de su actividad al Presidente de la República y al Parlamento".

13 Ley Orgánica N²011-333 de 2011.
} 
públicas independientes. Todo esto tampoco contribuye a aclarar la cuestión de la independencia de estas autoridades administrativas independientes.

\section{La independencia de las autoridades}

La voluntad de asegurar la independencia de dichas autoridades es, en sí mismo, algo inédito y excepcional. Pero es, precisamente, esa dinámica la que pude provocar discusión. Para sintetizarlo, podríamos decir que el debate se desarrolla en una doble vertiente. La primera, relativa a la naturaleza de esta independencia; La segunda, se relaciona con las funciones de esta independencia.

\section{a) Naturaleza de la independencia}

En cuanto al primer punto, es imprescindible discutir sobre la naturaleza de la independencia: ¿qué tipo de independencia debe asegurar el orden jurídico a esos organismos para que se pueda considerarles independientes stricto sensu? Sabemos que la independencia se aplica para todos los poderes y en todas las direcciones, sean económicas y políticas. Desde ese punto de vista, hay que tener en cuenta que frente a los intereses económicos considerables que conviven hoy en cada sociedad, en un mundo globalizado, en la comunidad internacional toda y frente a la solidez de las estructuras de los grupos privados, la independencia económica de esos organismos y de los miembros que las integran no puede ser ignorada. Hay que imaginar condiciones que permitan asegurar o garantizar de la mejor manera posible su autonomía frente a presiones financieras externas.

En relación con el poder político, dicha cuestión parece compleja y quizás lo sea aún más puesto que paradójicamente la necesidad de libertad que requiere el organismo en cuestión se contrapone al hecho de que la figura de la que estamos hablando, es al mismo tiempo parte de la misma administración. Para decirlo de otro modo, se pretende con la figura de la autoridad administrativa independiente crear una entidad cuyo origen surge como elemento interno o propio de la administración, es decir, como elemento de fuerza del poder político -lo que significa estar desprovisto de toda independencia frente a dicho poder- pero al mismo tiempo, dotándola de una característica excepcional -la independencia- que es, para decirlo de manera directa, contraria al espíritu que rige a la administración (al menos según la concepción que históricamente dominó la construcción del aparato administrativo francés). Este antagonismo lleva hasta el punto de que la pregunta podría ser la siguiente: ¿Puede una autoridad ser administrativa y tener a la vez un estatuto independiente?

Este interrogante, aunque parezca radical, no puede ser ocultado o ignorado y precisamente es para enfrentarlo que nacieron esas reflexiones a propósito del estatuto de esas autoridades. Es decir, se intentó imaginar elementos estatutarios capaces de propiciar garantías de independencia. Y desde este punto de vista, a través del régimen jurídico pueden ser las com- 
petencias, el estatus y el propio control, las palancas que permitan intentar responder a esta preocupación para borrar esa(s) contradicción(es).

Son evidentemente los textos que rigen el estatuto, las misiones y el funcionamiento que pueden ser instrumentos de esa independencia. Observamos que si bien hay similitudes entre las disposiciones en los textos que crean las autoridades administrativas independientes, se trata más de una inspiración común que de un régimen jurídico común. Dos vías han sido utilizadas hasta ahora: la de la independencia orgánica y la de la independencia funcional. Desde la óptica de la independencia orgánica, se nota que una de las características comunes de las autoridades administrativas independientes es su composición colegiada, que responde a dos exigencias: por un lado, la voluntad de equilibrio entre las influencias de las instancias de nominación - lo que constituye una garantía de independencia- y, por otro lado, asegurar una deliberación colectiva sobre asuntos sensibles o complejos, para una garantía de objetividad y de seriedad ${ }^{14}$. En cuanto, más precisamente, a las condiciones de designación de los miembros de las autoridades administrativas independientes, si no satisfacen las exigencias de independencia, las garantías estatutarias particulares pueden permitir compensarlo y así satisfacer dichas exigencias ${ }^{15}$.

Para responder a la misma preocupación, los miembros de las autoridades administrativas independientes están sujetos a varias obligaciones, y la disciplina reside en el autocontrol, es decir, de la asamblea respecto de sus propios miembros, lo que les da una verdadera independencia en el desempeño de sus funciones. La naturaleza y la amplitud de las obligaciones impuestas a los miembros de estas autoridades son muy diversas. Es necesario presentar brevemente estas obligaciones, por ser éstas tan importantes como lo es el periodo de los mandatos. En general, si bien las soluciones caben en un espectro común, son diferentes según las autoridades ${ }^{16}$. El mandato es, por supuesto, irrevocable. Sin embargo, sobre estos temas el legislador de 2017 no ha definido un marco general, la situación cambia según la importancia de las misiones de cada autoridad administrativa. La diferencia principal entre las diferentes autoridades reside en las formaciones dedicadas respectivamente a procesos penales y a las sanciones administrativas. La separación entre ambas formaciones se debe a las presiones de la jurispruden-

\footnotetext{
14 Pero el número de participantes en las asambleas es muy variable según las autoridades. Además, la idea de colegialidad tiene excepciones (Mediador de la energía, el Defensor de los derechos, el Supervisor general de los lugares de privación de las libertades).

15 Algunas de ellas están compuestas por magistrados de dos jurisdicciones: Conseil d'État, Cour de cassation y Cour des comptes. Con menos frecuencia diputados y senadores participan en unas autoridades (Comisión nacional informática y libertades o CNIL, Comisión de acceso a los documentos administrativos o CADA, Comisión nacional de control de las técnicas de inteligencia o CNCTR, por ejemplo). Pero lo más destacable es la participación en muchas autoridades administrativas independientes de representantes de la llamada sociedad civil. Es una verdadera innovación que subraya una evolución hacia la sociedad civil mediante personalidades designadas por su autoridad, su competencia o experiencia en un sector en particular.

16 La mayoría de los mandatos son de 5 a 6 años.
} 
cia de la Corte Europea de Derechos Humanos en relación con el principio de imparcialidad.

\section{b) Elementos funcionales de la independencia}

En cuanto a la independencia en su vertiente funcional, la autonomía de estos órganos también se ha de apreciar bajo la premisa de elementos funcionales. Entre estos elementos se encuentran los medios jurídicos, es decir, las competencias de estos órganos. Aquí tampoco existe ningún modelo establecido ni esquema predeterminado, de tal manera que solo se puede señalar el carácter amplio y diverso de las prerrogativas que disponen dichas autoridades. Ciertamente, es natural que esas autoridades ejerzan prioritariamente tareas administrativas. Sin embargo, algunas autoridades también disponen de prerrogativas casi jurisdiccionales. En la mayoría de los casos es la función de "regulación" que permite justificar el cúmulo de esta gama de medios por una institución encargada de pilotear con imparcialidad, eficiencia y flexibilidad un sector de dichas actividades. En numerosos casos, la autoridad se analiza más como una autoridad consultiva, al menos parcialmente, que como un órgano administrativo. Dicha autoridad puede de esta forma, proporcionar asesoramiento, dictar instrucciones, formular recomendaciones o realizar aclaraciones.

A estas prerrogativas se añade frecuentemente el hecho de que dichas autoridades disponen de poder reglamentario. Para resultar constitucionalmente admisible dicho poder no puede, primeramente, condicionar la existencia del poder reglamentario general que pertenece al Primer Ministro y, por otro lado, la habilitación legislativa bajo el fundamento del cual se otorga tiene que limitarse a medidas "limitadas tanto en su ámbito de aplicación como en su contenido"17. Diversos organismos como, por ejemplo, la CNIL, la Comisión de Sondeos o el Consejo Superior Audiovisual disponen de dicho poder reglamentario. El cúmulo del poder reglamentario y del poder de sanción no resulta fuente de dificultad, siempre que el poder de sanción se organice de modo de asegurar el respeto del derecho de defensa, el carácter contradictorio del procedimiento y la imparcialidad de la decisión.

A propósito del hecho de que las autoridades administrativas independientes dispongan de un verdadero poder de sanción administrativa, es decir, de un poder que afecte a la situación funcional y patrimonial de los destinatarios de dichas sanciones, la cuestión fue discutida y finalmente no ha sido admitida fácilmente. Sin embargo, es verdad que dicho poder tiende a generalizarse, aunque todas las autoridades administrativas independientes no disponen del poder de sanción sistemáticamente. En todo caso, ha de respetarse una doble reserva: si estas autoridades disponen de dicha facultad, es sólo en el límite exclusivo de las necesidades relativas al buen ejercicio de sus misiones, y la sanción pronunciada nunca puede desembocar en

17 Conforme con la Jurisprudencia del Consejo Constitucional Francés: Decisión N88-248 DC (1989). 
una medida privativa de libertad, que únicamente autoridades judiciales tienen el poder de tomar. Por otro lado, varias garantías procesales y de fondo enmarcan este poder administrativo represivo, contribuyendo a dotarlo de un cierto aspecto judicial ${ }^{18}$.

En todo caso, observamos que desde el punto de vista de la independencia, como ya era el caso de la definición, no hay una única solución o una respuesta única por parte del ordenamiento jurídico francés. Finalmente, se sigue buscando la homogeneidad o la unidad de la categoría.

\section{Una noción a la búsqueda de su unidad}

Dos caminos pueden ser imaginados o analizados hoy como vías para lograr una forma de unidad de la categoría de las autoridades administrativas independientes. Los dos nos ofrecen fundamentos igualmente robustos, sobre todo la vía legislativa, la que no consigue abarcar todas las entidades ya existentes (1), a pesar de que la existencia de unas formas de control, sobre todo jurisdiccional, aparezca mucho más como una vía conveniente y común a todos esos organismos, aunque no sea también totalmente satisfactoria (2).

\section{La unidad a través de la ley}

La Ley Orgánica del 20 de enero de $2017^{19}$, como la ley ordinaria del mismo día ${ }^{20}$ después de casi cuarenta años, pretendía cerrar las reflexiones, tanto parlamentarias como doctrinales, a propósito de esta figura que nació a principios de los ochenta bajo la apelación de "autoridad administrativa independiente". El esfuerzo principal consistía en una clarificación y racionalización de una categoría jurídica considerada, desde mucho tiempo, como faltante de coherencia. Ya en 2001, el Consejo de Estado proponía solamente una puesta en orden de la categoría y unas recomendaciones para tener en cuenta de la dificultad de juntar todas las autoridades existentes dentro de un único estatuto común. En aquella época, el informe del Alto Tribunal catalogó 34 organismos administrativos que podían ser calificados como autoridades administrativas independientes, sea por razón de la elección del legislador o del juez, sea por razón de sus características que eran conforme con los criterios identificados por el propio Consejo de Estado.

En 2015, en un nuevo informe del Senado ${ }^{21}$, se identificaron hasta 42 autoridades administrativas independientes y autoridades públicas independientes. $Y$ en los hechos, muchos organismos que no fueron necesariamente

\footnotetext{
18 Consejo constitucional, Décision N²000-433 DC (2000), considérant 50.

19 Ley Orgánica N²017-54 de 2017.

20 Ley $N^{\circ} 2017-55$ de 2017.

21 Un État dans l'État: canaliser la prolifération des autorités administratives indépendantes pour mieux les contrôler, Rapport fait au nom de la commission d'enquête sur le bilan et le contrôle de la création, de l'organisation, de l'activité et de la gestion des autorités administratives indépendantes, bajo la presidencia de Mme M.-H. Des Esgaulx, rapporteur M. J. Mézard, senadores, No 126 (2015-2016), 28 octubre de 2015.
} 
pensados en el momento de su creación como integrantes de esas categorías, sí lo fueron después de su aparición y a partir de la interpretación de la supuesta voluntad del legislador, almacenados en esta categoría; es decir, como si el Parlamento descubriera que había creado, sin saberlo, autoridades administrativas independientes. Desde este punto de vista, se podía considerar que las distintas autoridades fueran más de aparición aleatoria que el resultado de una estrategia global y reflexionada para transformar el modelo de administración francés.

Entonces, la voluntad del legislador de 2017 era la de realizar una labor de clasificación para limitar la categoría a unas entidades que comparten elementos comunes que son propios de un modelo de administración específico. Para lograr este objetivo, el trabajo, muy voluntarista, tenía la intención de utilizar la vía de la ley ordinaria y la vía orgánica para conseguir la reunión de las reglas comunes a esas autoridades dentro de un texto único. En este sentido, por un lado, la ley ordinaria se encarga de fijar un estatuto general para las autoridades administrativas independientes y para las autoridades públicas independientes; por el otro, la ley orgánica aparece imprescindible para fijar elementos que pertenecen a la reserva de competencia del legislador orgánico, es decir, sobre todo los elementos relativos a incompatibilidades con mandatos en colectividades territoriales de ultramar y de Nueva-Caledonia, con funciones dentro del Consejo Superior de la magistratura, del Consejo Económico Social y Medioambiental o con la función de magistrado o ciertos nombramientos por parte del Presidente de la República.

El principal objetivo es, entonces, armonizar el estatuto de las autoridades públicas independientes y de las autoridades administrativas independientes que figuran, de ahora en adelante, en una lista creada por la ley, sin que se clarifique la cuestión de sus atribuciones o las discusiones a propósito de eventuales fusiones o supresiones de tales autoridades. Pero, como se puede imaginar, esto no permitió aclarar todos los problemas y las preguntas jurídicas que ya habían sido identificadas. De tal forma que el Parlamento no logró la reunión de todos los elementos troncales y elementales de estas entidades diferentes. Con la preocupación de luchar contra la inseguridad jurídica que conlleva la existencia de tantas autoridades diversas, el legislador se encontró, finalmente, frente a la elección imposible entre las ventajas de un estatuto hecho a medida y las de un estatuto uniforme y único para todas.

Recordemos que en el año 2001, para el Consejo de Estado no parecía posible crear un estatuto único de autoridades administrativas independientes; pero esto no impedía el reconocimiento de unas reglas básicas comunes relativas a la garantía de su independencia y de su imparcialidad, dentro del espectro de las exigencias constitucionales y europeas. En 2017, el legislador se atrevió a usar la expresión de estatuto general, pero renunciando en compensación a ciertas reglas comunes, lo que significa que la elección fue a favor de la unidad a costa de un mínimo común denominador. Así, la ley reúne en un marco jurídico único autoridades administrativas y autoridades 
públicas independientes sin aclarar las relaciones entre esas dos entidades, cuando sabemos que las segundas cuentan con personalidad jurídica lo que pude suponer elementos estatutarios peculiares. De tal forma que la unidad dista mucho de ser perfecta y podría aun verse desestabilizada. Y, de forma bastante lógica, la ley contiene la enumeración limitativa de esas autoridades administrativas independientes y autoridades públicas independientes: solo 26 fueron consideradas como tales por el legislador que, al producir esta lista confiesa la imposibilidad de propiciar una definición operatoria. La ley concurre a la identificación de la noción sin poder cumplir totalmente con su ambición de cerrar la unidad de la materia. Quizás la existencia de control(es) sobre esas autoridades contribuye también a su armonización.

\section{La unidad a través del control}

Toda autoridad administrativa se encuentra sometida a diversas formas de control. Por supuesto, las autoridades administrativas independientes y las autoridades públicas independientes no escapan a esta evidencia. Pero, lo que quizás durante un tiempo fue propio de esas entidades es la presencia, dentro de esos controles, de un control jurisdiccional realizado en última instancia por un juez especial, es decir, el más alto juez de lo contencioso-administrativo francés, el Consejo de Estado. Así que, esta característica pudo funcionar, durante un tiempo, como criterio específico de identificación de esos organismos. La verdad es que, después de la reforma de 2017, la eficacia de este dato o índice no sigue siendo segura porque asistimos a una multiplicación de los tipos de control como a su diversificación, aun hasta el juez ordinario, es decir, el juez judicial.

En cuanto al tema del control, la primera observación que tenemos que hacer es que, en cuanto autoridades administrativas independientes, esos organismos no están sometidos al poder jerárquico. Es una evidencia; sin embargo, es importante recordarlo, al igual que hay que destacar que el poder de definir su propio reglamento interno tampoco está carente de significado, particularmente en el terreno del derecho de defensa y del procedimiento contradictorio.

En materia presupuestaria, si es difícil afirmar la existencia generalizada de control, es también difícil imaginar o concebir, dentro de un Estado unitario como es la República francesa, su ausencia absoluta. En efecto, con respecto a los recursos financieros, en los casos en los cuales las autoridades administrativas independientes carecen de personalidad jurídica, tampoco disponen de patrimonio alguno o de recursos propios susceptibles de otorgarles una autonomía financiera. Entendemos que, en esa configuración, dependen, en todo lo relativo a sus gastos, al organismo que se encarga de administrar sus finanzas. Entonces, en esta materia, la cualidad -para decirlo así- de su independencia será directamente relacionada con el espacio de libertad que se les deja en materia de decisión relativa al uso de los recursos que le corresponden. Sin embargo, cuando la ley las califica como autoridades públicas independientes o que son titulares de personalidad jurídica son, 
por supuesto, dotadas de un patrimonio propio, lo que disminuye la presión del control presupuestario que soportan que, empero, en ningún caso desaparece. Además, pueden beneficiarse de exenciones fiscales. No obstante, dicho lo cual, hay que resaltar que, aun cuando no disponen de personalidad jurídica propia, la ejecución de los gastos de las autoridades administrativas independientes nunca puede ser objeto de un control a priori, puesto que un control de este tipo constituiría la negación absoluta de toda autonomía presupuestaria.

Sobre el tema del control jurisdiccional al que son sometidas las autoridades administrativas independientes, tomando en cuenta el carácter administrativo de dichas autoridades, la competencia recae, en principio, en el juez contencioso administrativo. No obstante, aun en relación con este criterio, como lo hemos adelantado, observamos que, al contrario de lo que ocurrió durante un tiempo, no seguimos encontrando tampoco aquí el elemento que permite de manera absoluta y automática identificar o asegurar la unidad de la categoría. En efecto, existen ahora ciertos casos en los cuales el juez judicial es competente, especialmente en materia de regulación económica. Así, en dicho caso y tomando en cuenta el imperativo "de buena administración de la justicia", resulta necesario definir bloques competenciales que justifican que se derogue la competencia de principio del juez administrativo, evitando que se multipliquen los casos en los que la aplicación de las reglas generales desemboque en una competencia compartida entre juez judicial y juez administrativo ${ }^{22}$.

En todo caso, el grado de control ejercido por el juez varía en función de la gravedad de la decisión tomada por la autoridad administrativa. Así, el Consejo de Estado ha estimado, en primer lugar, que los recursos contra las sanciones administrativas se han de juzgar en plano contencioso ${ }^{23}$. De modo igualmente muy general, el Consejo ha hecho progresar para todas las sanciones administrativas la intensidad de su control de la adecuación entre le gravedad de la sanción y la gravedad de los hechos reprochados, y procede ahora a un control entero de proporcionalidad ${ }^{24}$. Con respecto a las medidas administrativas ordinarias -que no tienen el carácter de sanciones- que llegan hasta la jurisdicción administrativa en base a los recursos de anulación, el juez aplica su jurisprudencia clásica que consiste en distinguir los casos en los cuales la autoridad ejerce un poder discrecional (para los cuales el control es restringido) y los casos en los cuales la autoridad se sitúa en situación de competencia vinculada (compétence liée).

Además, la ley del 20 de enero de 2017 reforzó dos controles: el del Parlamento y el del Gobierno sobre esas autoridades. Así, cada autoridad independiente, sea administrativa o pública, tiene que preparar cada año,

\footnotetext{
22 Se puede ver y leer la famosa decisión del Consejo Constitucional Nº 86-224 DC (1987).

23 CE, ass., 16 févr. 2009, Société Atom.

24 CE, 29 mai 2009, N³10356, Société Laboratoires Mayoli Spindler.
} 
antes del 1 de junio, un informe sobre su actividad para dar cuenta del ejercicio de sus misiones y de sus recursos, informe a destinación del Parlamento, pero también del gobierno. De tal modo que, para concluir, podemos observar que las leyes de enero del 2017, si bien no aportan ningún cambio fundamental, se inscriben en la vía de lograr una clarificación de la noción y del estatus de las autoridades administrativas independientes. Así, reconocen expresamente la calidad de autoridades administrativas independientes o de autoridades públicas independientes a 26 autoridades, de tal manera que pareciera que dicha lista tiene carácter limitativo y que, de aquí en adelante, el criterio único de identificación se fundamenta en la cualificación expresa por el legislador. A partir de esta fecha, esos dos tipos de autoridades administrativas se pueden definir como aquellas autoridades a la cuales se les aplica el estatus general previsto por la ley orgánica y la ley ordinaria, todas de 2017. Exceptuando las imperfecciones que hemos identificado, tienen el mérito de enmarcar fuertemente para el futuro la creación de nuevas autoridades administrativas independientes, de someterlas a reglas estatutarias que, sin ser idénticas, podrán ser similares y de progresar en el esfuerzo de identificación de lo que sigue siendo, en parte, un objeto original del modelo administrativo francés.

\section{Bibliografía citada}

CE (2009). Société Atom. Disponible en: https://www.fallaitpasfairedudroit.fr/images/files/ Droit\%20administratif/21\%20-\%20REP/Controle_Sanctions_administratives_-_CE__13112013_-_Dahan.pdf

CONSEIL D'ÉTAT (2001). Rapport public 2001. Les autorités administratives indépendantes, Études et Documents No 52. Disponible en: http://www.conseil-tat.fr/content/download/368/1132/version/1/file/rapport-public2001.pdf

CONSEIL D'ETAT (2009). Société Laboratoires Mayoli Spindler. Disponible en: http://www. monod-colin-stoclet.com/sites/default/files/pdf/lettre_droit_pharma_octobre_2009.pdf

CONSEJO CONSTITUCIONAL (1987). Loi transférant à la jurisdiction, judiciaire le contentieux des decisions du Conseil de la concurrence. Disponible en: https://www.conseil-constitutionnel.fr/decision/1987/86224DC.htm

DosièRE, René et VANNESTE, Christian (2010). Les autorités administratives indépendantes: pour une indépendance sus la garantie du Parlement. Disponible en: http://www.assemblee-nationale.fr/13/rap-info/i2925-ti.asp

GÉLARD, Patrice (2006). Les autorités administratives indépendantes: évaluation d'un objet juridique non identifié. Disponible en: https://www.senat.fr/notice-rapport/2005/r05404-2-notice.html

SÉNAT (2015). Un État dans l'État: canaliser la prolifération des autorités administratives indépendantes pour mieux les contrôler, Rapport fait au nom de la commission d'enquête sur le bilan et le contrôle de la création, de l'organisation, de l'activité et de la gestion des autorités administratives indépendantes. Disponible en: http://www.senat. $\mathrm{fr} / \mathrm{rap} / \mathrm{r} 15-126-1 / \mathrm{r} 15-126-18 . h \mathrm{tml}$

\section{Normativa citada}

Ley $N^{\circ} 78-17$, Relativa a la informática, a los ficheros y a las libertades. Publicado 6 enero 1978.

Ley $N^{\circ}$ 2003-706, de Seguridad Financiera que fusiona el Consejo de los mercados financieros, la Comisión de las Operaciones de Bolsa y el Consejo de disciplina de la gestión financiera. Publicado 1 agosto 2003 
Ley N ํ 2004-810, relativa al Seguro Médico. Publicado 13 agosto 2004.

Ley $N^{\circ} 2006-405$, relativa a la lucha contra el dopaje y a la protección de la salud de los deportistas. Publicado 5 abril 2006.

Ley Constitucional № 2008-724, de modernización de las Instituciones de la Quinta República. Publicado 23 julio 2008.

Ley $N^{\circ}$ 2009-669, sobre difusión y protección de la creación en Internet. Publicado 12 junio 2009.

Ley $\mathrm{N}^{\circ}$ 2009-1503, relativa a la organización y a la regulación de los Transportes Ferroviarios y comportando diversas disposiciones relativas a los transportes. Publicado 8 diciembre 2009.

Ley Orgánica N²011-333, relativa el Defensor de los derechos. Publicado 29 marzo 2011.

Ley No 2015-990 para el crecimiento, la actividad y la igualdad de oportunidades económicas. Publicado 6 de agosto 2015.

Ley Orgánica N²017-54, relativa a las Autoridades Administrativas Independientes y a las Autoridades Públicas Independientes. Publicado 20 enero 2017.

Ley $N^{\circ}$ 2017-55, sobre el Estatuto General de las Autoridades Administrativas Independientes y de las Autoridades Públicas Independientes. Publicado 20 enero 2017.

\section{Jurisprudencia citada}

Consejo Constitucional Francés: Décision N 88-248 DC del 17 de enero de 1989.

Consejo Constitucional: Décision N²000-433 DC del 27 de julio de 2000, considérant 50. 